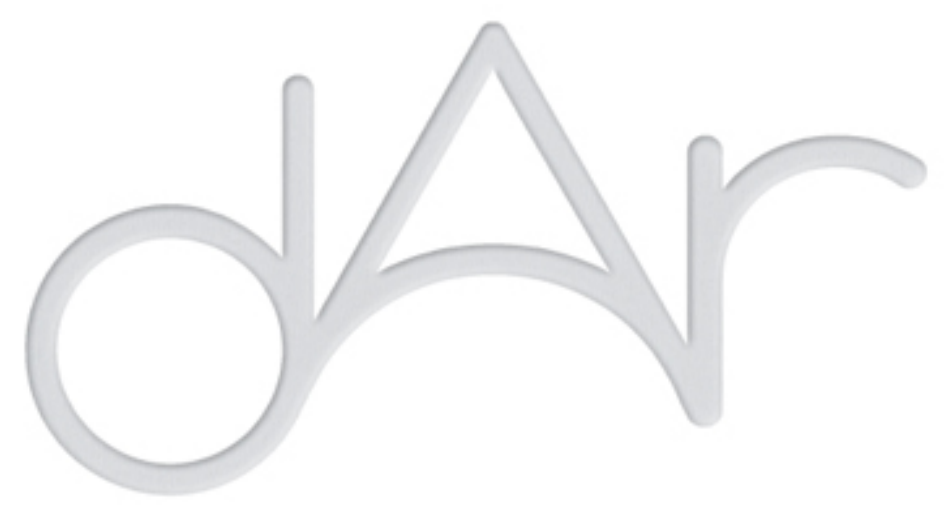

La prospective territoriale au service du patrimoine oasien: cas des oasis de Tafilalet

Autor(es): $\quad$ Ouhajou, Lakbir; Lmariouh, Ali

Publicado por: Imprensa da Universidade de Coimbra

URL persistente:

URI:http://hdl.handle.net/10316.2/44423

DOI:

DOI:https://doi.org/10.14195/2182-844X_5_2

Accessed : $\quad$ 26-Apr-2023 08:16:32

A navegação consulta e descarregamento dos títulos inseridos nas Bibliotecas Digitais UC Digitalis, UC Pombalina e UC Impactum, pressupõem a aceitação plena e sem reservas dos Termos e Condições de Uso destas Bibliotecas Digitais, disponíveis em https://digitalis.uc.pt/pt-pt/termos.

Conforme exposto nos referidos Termos e Condições de Uso, o descarregamento de títulos de acesso restrito requer uma licença válida de autorização devendo o utilizador aceder ao(s) documento(s) a partir de um endereço de IP da instituição detentora da supramencionada licença.

Ao utilizador é apenas permitido o descarregamento para uso pessoal, pelo que o emprego do(s) título(s) descarregado(s) para outro fim, designadamente comercial, carece de autorização do respetivo autor ou editor da obra.

Na medida em que todas as obras da UC Digitalis se encontram protegidas pelo Código do Direito de Autor e Direitos Conexos e demais legislação aplicável, toda a cópia, parcial ou total, deste documento, nos casos em que é legalmente admitida, deverá conter ou fazer-se acompanhar por este aviso.

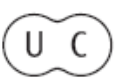



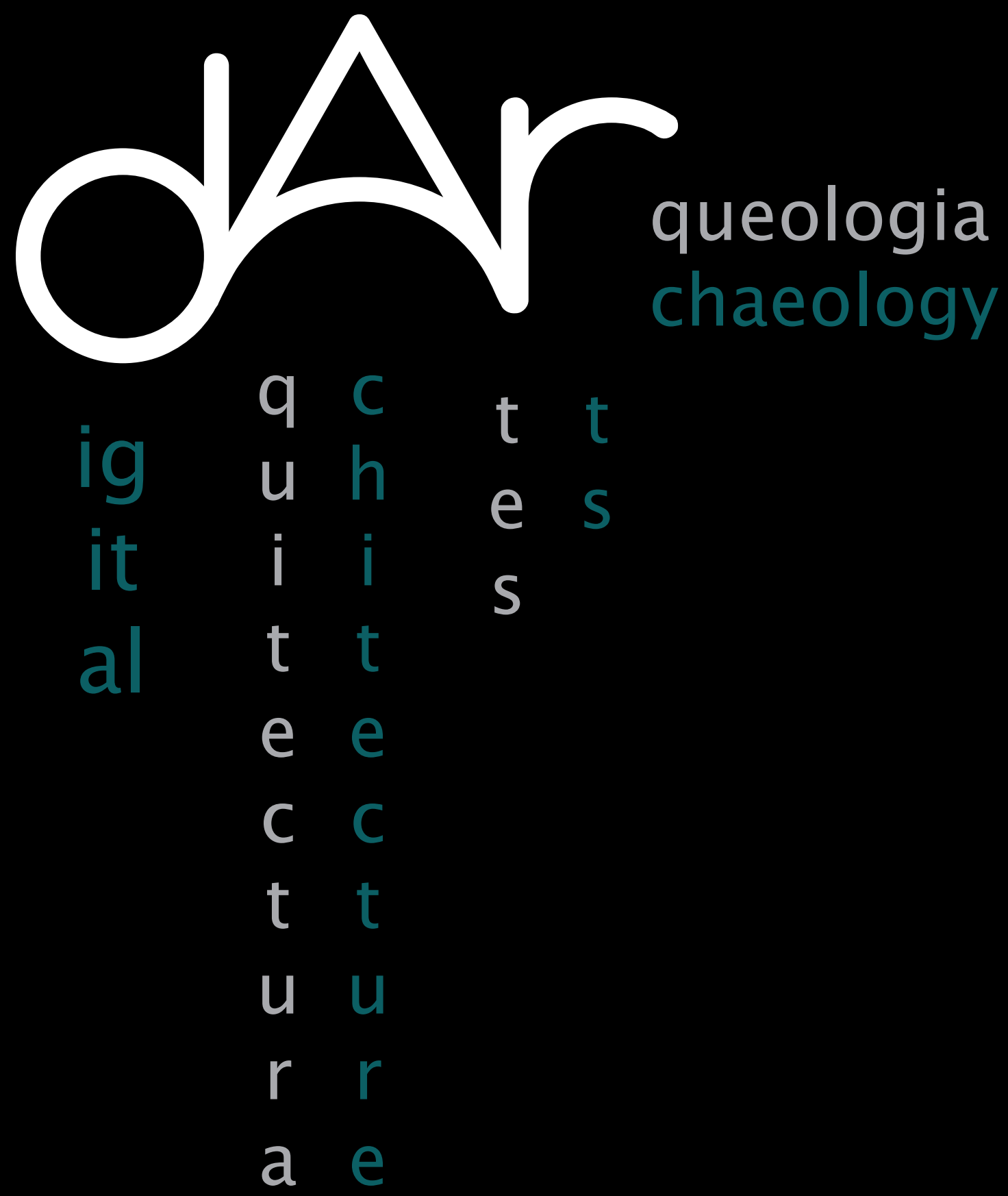

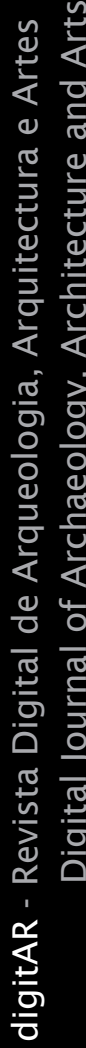




\title{
La Prospective Territoriale au service du patrimoine oasien: CAS DES OASIS DE TAFILALET
}

\author{
Dr. Lakbir Ouhajou, Ali Lmariouh \\ Faculté des Lettres et des Sciences Humaines, \\ Agadir
}

\section{Résumé}

L'article propose une démarche de prospective comme outil novateur pour accompagner la sauvegarde de ce patrimoine vulnérable que représentent les oasis. Ce cheminement méthodologique d'innovation sociale, outil de l'ingénierie territoriale, ambitionne de concevoir une vision de développement territorial pour les oasis de Tafilalet. Il s'agit de proposer une série de futurs souhaitables, qui vont dans le sens de renforcer la résilience de ces territoires aux changements climatiques, et de rompre par la même avec les tendances territoriales actuelles.

\section{Introduction}

Au Maroc, le développement des territoires oasiens suscite depuis plusieurs années l'attention des pouvoirs publics. Ceux-ci ont initié de nombreux programmes et projets, notamment la stratégie de développement et d'aménagement des oasis, ainsi que des programmes spécifiques par zones oasienne (Tafilalet, Draa, Figuig...). Toutefois, l'état de ces territoires prête toujours à interrogation. De nombreuses problématiques telles que l'ensablement, la désertification, la régression du niveau de vie et l'augmentation de la pauvreté économique restent à l'ordre du jour. Elles interpellent entre autre la manière avec laquelle les acteurs réfléchissent la planification de ces territoires, obéissant généralement à une logique courtermiste se basant sur la projection et la prévision (Lmariouh, 2014). Or, selon la littérature, une planification territoriale réussie passe nécessairement par une réflexion sur le long terme, à travers une démarche prospective qui s'inscrit dans une logique proactive, longtermiste intégrant les ruptures. Cette 
ingénierie territoriale novatrice questionne les trajectoires de développement sur lesquelles pourraient se baser le futur du territoire en question. Ce qui n'est pas le cas du Maroc. Dans cette lignée, nous ambitionnons de questionner le rôle de la démarche prospective comme innovation sociale, dans le processus de sauvegarde de ce patrimoine national que sont les oasis, en proposant un cheminement méthodologique dont le fondement se trouve à la croisée de plusieurs disciplines scientifiques, dont notamment les sciences de gestion, la géographie, l'économie et la sociologie.

\section{Le Tafilalet. un territoire vulnerable a potentialites variees et menacees}

Le Tafilalet (Province d'Errachidia) est situé au sud-est du royaume du Maroc. La province est marquée par un climat aride et abrite une population avoisinant les 420 milles habitants, en grande partie rurale. Sur le plan des activités économiques, le Tafilalet reste un territoire principalement agricole avec une spécificité de production de dattes. En effet, le Tafilalet abrite $29 \%$ de la superficie réservée au palmier dattier au niveau national. Cette province participe en outre à hauteur de $27 \%$ dans la production nationale de dattes. D'autre part, la ressource en eau y est menacée par la persistance de sécheresses récurrentes dues principalement au changement climatique, mais aggravée par la mauvaise gestion, la persistance des pratiques culturales inadaptées et l'accroissement de la population.

\section{La prospective territoriale et la methode proposee}

L'idée centrale inhérente à la prospective est que l'avenir n'est pas une fatalité, qu'il se construit et qu'il est moins à découvrir qu'à inventer. A cet effet, il reste nécessaire de faire preuve d'anticipation, sans elle, les urgences ne laissent guère de marges de manœuvre [Durance, Godet, Mirénowicz, Pacini, (2007)]. La prospective s'efforce de réduire l'incertitude face à l'avenir, de décrypter et d'envisager collectivement des futurs possibles, de faire émerger la vision d'un futur souhaitable, ainsi que la trajectoire pour y parvenir.

La montée en puissance des démarches prospectives dans le processus d'élaboration des politiques de développement a été motivée par des besoins réels de visibilité ainsi que par l'échec des approches traditionnelles. La prospective territoriale peut être définie comme étant une démarche d'intelligence collective et d'aide à la décision qui éclaire pour définir des pistes exploratoires et imaginer des cheminements vers des transformations 


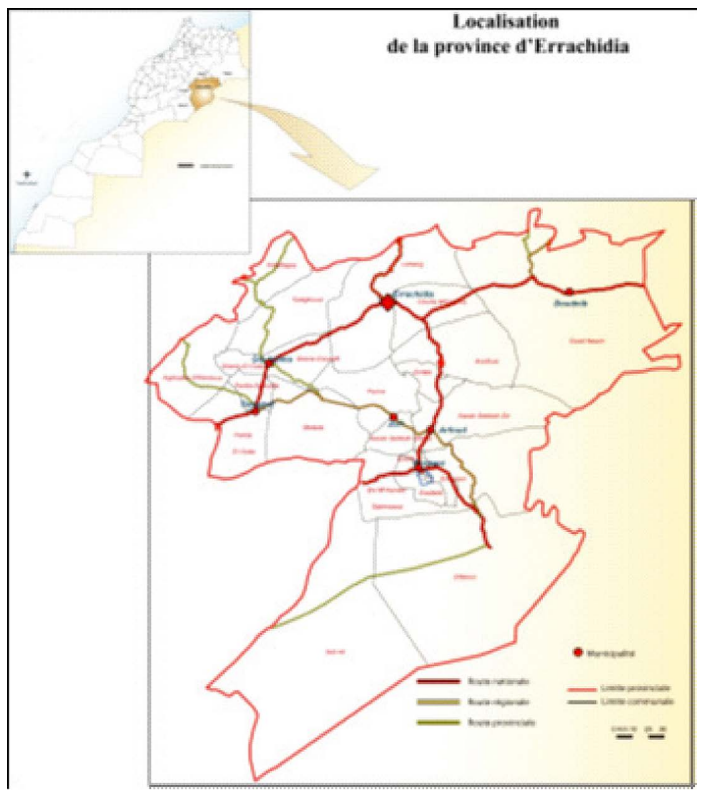

Carte 1 - Localization geographique de la province d' Errchidia Souce: Rapport “Tafilalet 2040”, MUAT-Rabat, 2014 sur les territoires (GPT, 2007). En d'autres termes, l'avenir d'un territoire devrait être le produit d'une réflexion basé sur un diagnostic territorial et formalisé dans le cadre d'un projet de territoire.

Pour ce faire, Il n'y a pas de pratiques universelles pour esquisser l'avenir d'un territoire. Néanmoins, quelques méthodes sont plus mobilisées que d'autres. L'idée principale de la plupart d'entre elles est d'opérer un aller-retour entre le présent et le futur à travers une série de décomposition/recomposition et de croisements qui feront appel, tout le long $\mathrm{du}$ processus, à l'imagination et à

l'intelligence des acteurs territoriaux. La méthode avancée dans le cadre de cet article, ambitionne de proposer un cheminement méthodologique prospectif qui a pour objectif de mettre le long terme au service du développement territorial.

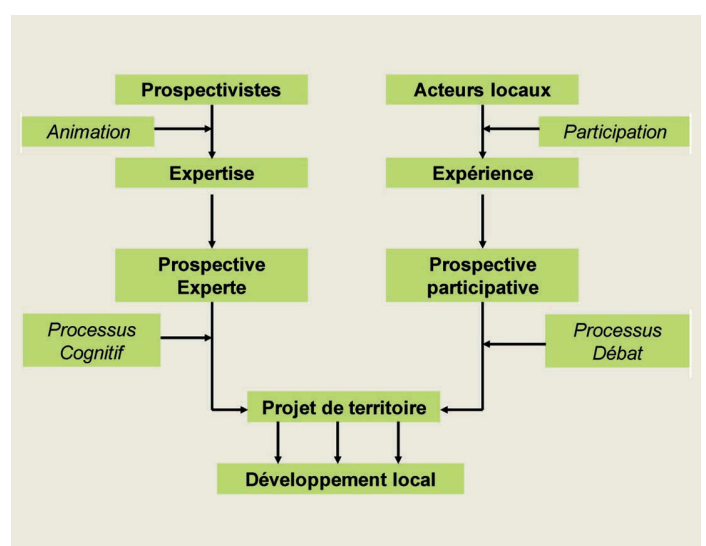

Fig. 1 - Alchimie entre l'expertise et l'experience
La démarche méthodologique proposée s'inscrit dans le sillon de la prospective experte et exploratoire :experte se basant en premier lieu sur la connaissance du terrain, le vécu et les accumulations des acteurs locaux ainsi que la littérature scientifique et techniques réalisés dans ce sens ; exploratoire, cherchant à savoir de quoi demain sera fait, nous partons du présent pour explorer des hypothèses 
de changements à long terme et leurs conséquences, des futurs possibles et des futurs souhaitables.

Il s'agit de s'ouvrir sur les futurs possibles en balisant les différentes configurations qui peuvent se produire (prospective exploratoire), et ensuite travailler sur quelques futurs souhaitables en détaillant les tenants et aboutissants qui doivent permettre d'énoncer et d'élucider les enjeux stratégiques pour l'avenir, susceptibles d'inspirer les changements de politiques et d'action (prospective normative). Enfin, il s'agira de revenir vers les acteurs et surtout les décideurs pour présenter les résultats du travail afin qu'ils puissent opérer des choix. Cette démarche de travail est orientée dans le sens de permettre au maître d'ouvrage, instigateur de l'exercice de prospective, d'opérer un choix parmi plusieurs propositions d'actions émanant des futurs possibles.

Pour offrir au Tafilalet une vision de développement territorial, l'idée principale n'étant pas de faire dans l'exhaustivité, l'exercice ne prétendra pas traiter tous les domaines et secteurs d'activité opérant sur le territoire. Il s'agit de se focaliser sur quelques thématiques clés autour desquelles se jouera le développement futur du territoire de Tafilalet. Concrètement, la méthode proposée consiste à mettre le développement territorial au centre du processus de réflexion, et procéder à travers des opérations de décomposition/recomposition à réaliser trois phases bien distinctes :

A. Faire ressortir les figures de développement par enjeu stratégique ;

B. Croisement des figures de développement et détail des futurs possibles ;

C. Choix des futurs souhaitables et caractéristiques principales.

2.1. Phase 1: ressortir les figures de développement par enjeu stratégique Il s'agit d'opérer un croisement entre les enjeux stratégiques et les filtres spatio-temporels. L'objectif de cette première phase est de faire passer les enjeux stratégiques, autour desquels se basera le développement futur de Tafilalet, par des filtres qui incarnent trois postures de changements.

\subsubsection{Les filtres spatio-temporels}

Les filtres spatio-temporels, au nombre de trois, renseigne sur éléments suivants :

1. Filtre 1: Les invariants. Qu'est ce qui pourrait ne pas changer?

2. Filtre 2 : Les tendances lourdes et les faits porteurs. Qu'est-ce qu' on peut déjà prévoir pour demain?

3. Filtre 3 : Les ruptures et les discontinuités. Qu'est ce qui demeurera imprévisible? 


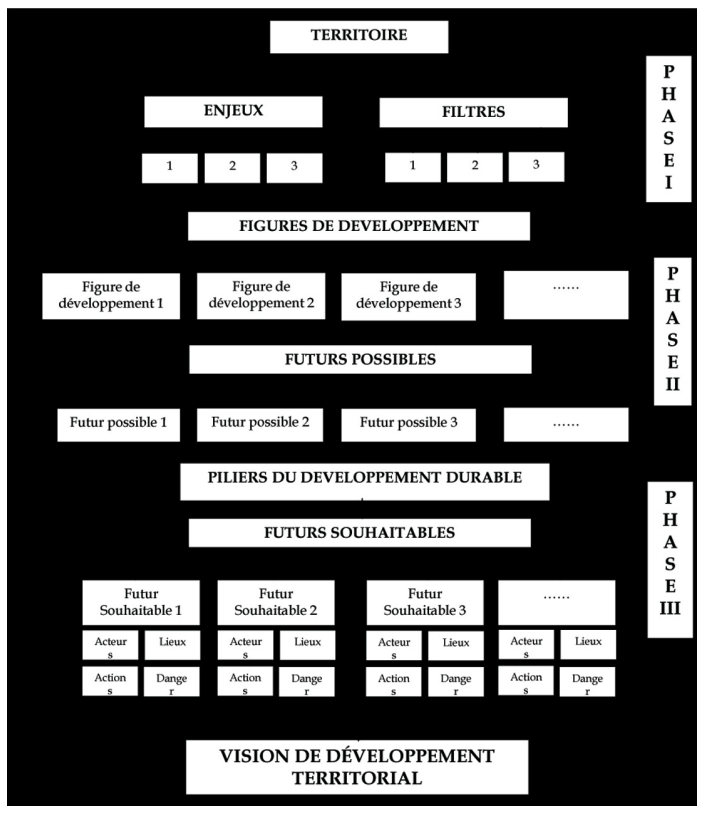

Fig. 2 - Enchainement méthodologique proposé

\subsubsection{Les enjeux stratégiques}

Dans le Tafilalet, le diagnostic élaboré nous renseigne sur un certain nombre de facteurs limitant avec lesquels devra se conjuguer chacune des propositions de développement : la ressource en eau et la protection de l'environnement. Le développement de la zone se basera, à notre humble avis, autour de trois enjeux stratégiques : le développement agricole, le développement touristique, et le développement urbain.

a) Enjeu 1: le développement Agricole

Dans le Tafilalet se développe une agriculture oasienne en évolution, utilisant des systèmes d'irrigation de plus en plus innovant (pompe solaire, goute à goute), et qui connait une diversification de plus en plus grande (palmier-dattier, arbres fruitiers, plantes aromatiques et médicinales). Toutefois, cette tendance est exposée à deux facteurs contraignants ou limitatifs en l'occurrence l'eau en premier lieu et les moyens financiers en second lieu. Actuellement, la zone connait une pénurie d'eau vue la faiblesse des précipitations durant ces trois dernières années. Par rapport à la SAU, le Schéma Régional d'Aménagement du Territoire de Meknes-Tafilalet nous renseigne que le Tafilalet renferme des densités de 800 à 900 habitants $/ \mathrm{km}^{2}$. Il est évident que la terre ne peut pas nourrir convenablement 8 à 10 personnes par hectare. Les exploitations sont très petites et très morcelées. Le Plan Provincial de Développement Rural ajoute que l'agriculture représente $90 \%$ des activités économiques de la province, et emploie environ $60 \%$ de la population active dans le périmètre irrigué des oasis. La Stratégie Nationale d'Aménagement et de Développement des Oasis pour sa part, nous renseigne que la phoeniculture est l'ossature de l'activité agricole dans les oasis, malgré les contraintes climatiques caractérisant la zone. 
b) Enjeu 2: le développement touristique

Le Tafilalet jouit d'une infrastructure d'hébergement assez suffisante répartie entre les principaux pôles d'attraction qui sont Erfoud, Errachidia, Rissani et Goulmima. L'activité touristique connait une très forte concentration au niveau de l'axe Erfoud-Merzouga en passant par Rissani ( $81 \%$ de la capacité d'hébergement).Le Produit touristique offert par le territoire se caractérise par la diversité de ses composantes : (tourisme de désert, tourisme des oasis, tourisme culturel, tourisme de montagne, thermalisme et cures).Sur cette base, la vision $2020 \mathrm{du}$ ministère du tourisme propose désormais que le Tafilalet, fasse partie de la région touristique «Atlas et Vallée». Le territoire se positionne dans l'écotourisme et le développement durable. Des ambitions bien déterminées à l'horizon 2020 pour diversifier l'offre touristique à travers la programmation de différents projets susceptibles d'attirer une clientèle plus variée «Ex : Tourisme de luxe à Merzouga, Club équestre à Meski, Escalade à Amellagou...».

c) Enjeu 3: le développement urbain

Seule véritable ville, Errachidia atteint à peine 80.000 habitants. Elle est issue d'un grand souk traditionnel renforcé par un poste militaire et transformé après 1975 en principale ville administrative et militaire. C'est essentiellement une ville d'état, animée par les dépenses publiques, autour desquelles s'est développée une offre de services à la population locale et régionale. Sa position marginale est à la fois un handicap pour le développement d'activités économiques intégrées dans le système productif national, et un avantage, car elle justifie l'effort public sans lequel la ville n'aurait pas connu le développement rapide qui a été le sien. La ville est composée de plusieurs noyaux développés à partir de ksour.Les autres localités urbaines sont de petites villes au débouché des vallées du versant sud de l'Atlas (Tinejdad sur l'oued Ferkla, Goulmima sur le Gheris, Boudnib sur le Guir, Er-Rich). Ces derniers sont des centres locaux intéressants mais dont la fonction limite le rayonnement. Ils subissent actuellement une augmentation de la population venant des parties supérieures des bassins fluviaux. Enfin, Erfoud est une petite bourgade née de l'activité touristique. Le réseau urbain est donc faible, et ne joue pas le rôle d'entraînement attendu, et le développement ne peut être conçu en l'absence d'un réseau urbain solide, cohérent et compétitif. En matière d'urbanisme, il y a lieu de noter le retard enregistré dans l'élaboration des PA ainsi que du SDAU de la vallée du Ghris. En somme, l'avenir de Tafilalet est tracé 
par un développement tendanciel autour de 3 polarités urbaines : le Grand Errachidia, Erfoud-Errissani et Goulmima-Tinejdad.

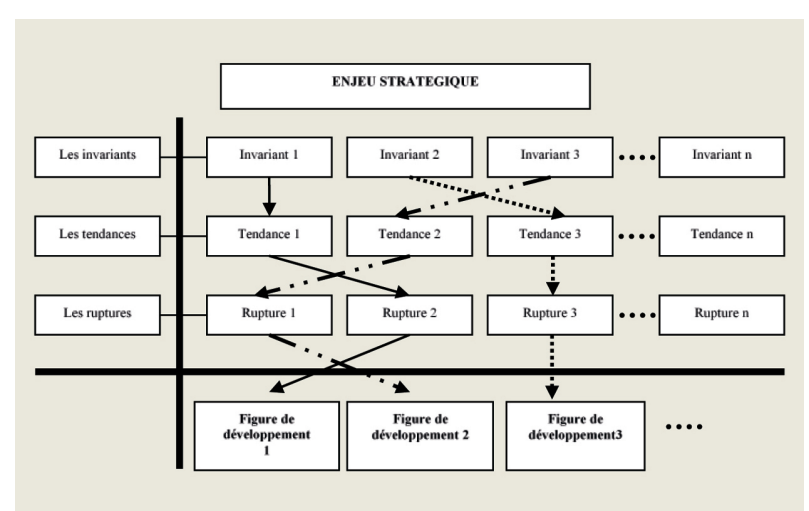

Fig. 3 - Les figures de dévelopement par enjeu

\subsubsection{Résultat du croise- ment}

Il s'agit de répondre à la question : Quelles images du futur peut-on imaginer ? Durant cette phase, on procèdera par enjeu stratégique, à la recherche des combinaisons les plus ou moins cohérentes entre les invariants, les tendances et les ruptures. Ces croisements représenteront les possibles figures de développement. L'exercice a fait ressortir deux configurations pour chaque enjeu.

Le résultat est présenté ci-dessous :

Pour le développement touristique

- Tourisme 1 (T1-Tourisme de niche) : Tafilalet est une destination touristique à reconfirmer ;

- Tourisme 2 (T2-Tourisme diversifié) : Tafilalet est une destination touristique à réinventer.

Pour le développement agricole

- Agriculture 1 (A1-gestion de la rareté) : Tafilalet s'inscrit dans une agriculture de la gestion de la rareté ;

- Agriculture 2 (A2-agriculture industrielle) : Tafilalet développe une agriculture industrialisée.

Pour le développement urbain :

- Urbain 1 (U1-urbanisme tendanciel) : Tafilalet avec un réseau urbain organisé en trois pôles ;

- Urbain 2 (U2-Réseau attractif) : Urbain 2 : Tafilalet avec un réseau urbain attractif. 


\section{Le résultat de cette première phase se présente ainsi :}

\begin{tabular}{|c|c|c|c|c|c|}
\hline \multicolumn{5}{|c|}{ DEVELOPPEMENT TOURISTIQUE } \\
\hline Invariants & Poids du secteur & \multicolumn{2}{|c|}{ Potentiel naturel et culturel important } & $\begin{array}{l}\text { Présence d'acteurs institutionnels } \\
\text { (délégation, SMIT, CPT..) }\end{array}$ \\
\hline Tendances & $\begin{array}{c}\text { Vision } \\
\text { Tourisme 2020 }\end{array}$ & Projet oasis sport & changements climatiques & $\begin{array}{c}\text { Développement du } \\
\text { tourisme de niche }\end{array}$ & $\begin{array}{c}\text { Crise sociale } \\
\text { accentuée }\end{array}$ \\
\hline Ruptures & $\begin{array}{c}\text { Régionalisation } \\
\text { avancée }\end{array}$ & $\begin{array}{c}\text { Création de l'Agence de } \\
\text { développement touristique }\end{array}$ & $\begin{array}{c}\text { Perte d'identité et } \\
\text { d'authenticité (disparition du } \\
\text { patrimoine bâti) }\end{array}$ & $\begin{array}{c}\text { Dégradation des } \\
\text { ressources }\end{array}$ & Aléas / crises \\
\hline \multirow{2}{*}{$\begin{array}{c}\text { Figures de } \\
\text { développement }\end{array}$} & $\begin{array}{l}\text { Tourisme 1: Tafilalet = destination touristique (fort potentiel naturel et culturel) à reconfirmer, évoluant dans } \\
\text { le tourisme de niche. Et dans lequel les autres types de tourismes se développent à leurs rythmes. } \\
\text { Danger : opérateurs s'éloignant de plus en plus de l'originalité et de l'authenticité du territoire. }\end{array}$ \\
\cline { 2 - 6 } & $\begin{array}{l}\text { Tourisme 2 : Tafilalet = destination touristique à réinventer : à la rechercher d'autres types de tourisme (de masse, } \\
\text { d'affaires, scientifique, médical...) et d'activités touristiques (récréatives, sportives...) à développer. Et dans lequel le } \\
\text { tourisme de niche devient à activité secondaire. Danger : dégradation de l'environnement, perte d'identité... }\end{array}$ \\
\hline
\end{tabular}

Tableau 1 - Les figures de dévelopement pour le dévelopement touristique

\begin{tabular}{|c|c|c|c|c|c|}
\hline \multicolumn{6}{|c|}{ DEVELOPPEMENT AGRICOLE } \\
\hline \multirow[t]{2}{*}{ Invariants } & $\begin{array}{l}\text { Poids du } \\
\text { secteur }\end{array}$ & \multicolumn{2}{|c|}{ Atomisation de la propriété foncière } & $\begin{array}{l}\text { Prolifération de la maladie } \\
\text { « Bayoud » }\end{array}$ & $\begin{array}{l}\text { structure foncière } \\
\text { complexe }\end{array}$ \\
\hline & \multicolumn{3}{|c|}{ Rareté des ressources hydriques } & \multicolumn{2}{|c|}{ Structures institutionnelles existantes } \\
\hline \multirow{2}{*}{ Tendances } & $\begin{array}{l}\text { Crise sociale } \\
\text { accentuée }\end{array}$ & $\begin{array}{l}\text { Changements } \\
\text { climatiques }\end{array}$ & $\begin{array}{l}\text { Approche genre } \\
\text { incontournable }\end{array}$ & $\begin{array}{c}\text { Organisation des agriculteurs } \\
\text { dans un statut professionnel }\end{array}$ & $\begin{array}{c}\text { Piétinement urbanistique } \\
\text { sur les terres agricoles }\end{array}$ \\
\hline & $\begin{array}{l}\text { Valorisation } \\
\text { des dattes }\end{array}$ & $\begin{array}{l}\text { Gestion de la } \\
\text { rareté de l'eau }\end{array}$ & $\begin{array}{l}\text { Plan Maroc vert, } \\
\text { stratégie ANDZOA }\end{array}$ & Extension agricole hors oasis & Sur-pompage \\
\hline \multirow[t]{2}{*}{ Ruptures } & \multicolumn{2}{|c|}{$\begin{array}{c}\text { Création Agence Nationale des } \\
\text { PAM }\end{array}$} & $\begin{array}{l}\text { Savoir-faire en } \\
\text { déperdition }\end{array}$ & Maladies phoenicicoles & Invasion acridienne \\
\hline & \multicolumn{2}{|c|}{ Abandon des oasis } & \multicolumn{2}{|c|}{ Appauvrissement des sols } & Nouveaux barrages \\
\hline \multirow{2}{*}{$\begin{array}{c}\text { Figures de } \\
\text { développement }\end{array}$} & \multicolumn{5}{|c|}{$\begin{array}{l}\text { Agriculture 1: Tafilalet = Agriculture (PAM, produits locaux et élevage) s'inscrivant dans la gestion de la } \\
\text { rareté avec encouragement de la transmission des savoir-faire (formation) ainsi qu'un statut foncier souple et une } \\
\text { pression urbanistique maitrisée. Et à coté de laquelle le développement d'une agriculture industrielle maîtrisée. } \\
\text { Danger : abandon des oasis. }\end{array}$} \\
\hline & \multicolumn{5}{|c|}{$\begin{array}{l}\text { Agriculture 2: Tafilalet = Agriculture à haute valeur ajoutée (industrialisée), en parallèle oublier l'agriculture } \\
\text { traditionnelle qui devient dans cette perspective nostalgique. Danger : extension hors oasis, intensif et sur-pompage, } \\
\text { accélération du tarissement de la ressource, concurrence déloyale. }\end{array}$} \\
\hline
\end{tabular}

Tableau 1 - Les figures de dévelopement pour le dévelopement agricole

\begin{tabular}{|c|c|c|c|c|c|}
\hline \multicolumn{6}{|c|}{ DEVELOPPEMENT URBAIN } \\
\hline \multirow[t]{2}{*}{ Invariants } & Errachidia =ville d'état & \multicolumn{2}{|c|}{ Absence des normes de construction en pisé } & \multicolumn{2}{|c|}{$\begin{array}{l}\begin{array}{l}\text { Présence des institutions en charge du } \\
\text { dossier }\end{array} \\
\end{array}$} \\
\hline & \multicolumn{2}{|c|}{ Existence d'un réseau urbain } & \multicolumn{3}{|c|}{ Absence d'opérateur pour le patrimoine } \\
\hline \multirow[b]{2}{*}{ Tendances } & $\begin{array}{l}\text { Urbanisation galopante } \\
\text { (éclatement) }\end{array}$ & $\begin{array}{c}\text { Généralisation des } \\
\text { documents d'urbanisme }\end{array}$ & \multicolumn{2}{|c|}{$\begin{array}{c}\text { Accentuation du caractère } \\
\text { touristique de la ville d'Errachidia }\end{array}$} & $\begin{array}{l}\text { Prolifération de l'habitat } \\
\text { non réglementaire }\end{array}$ \\
\hline & $\begin{array}{l}\text { Système d'assainissement } \\
\text { insuffisant }\end{array}$ & $\begin{array}{l}\text { Perte d'identité } \\
\text { architecturale }\end{array}$ & $\begin{array}{c}\text { Généralisation de } \\
\text { la mise à niveau } \\
\text { urbaine }\end{array}$ & Exode rural & Réhabilitation des Ksour \\
\hline Ruptures & $\begin{array}{l}\text { Dégradation d'espaces } \\
\text { naturels }\end{array}$ & $\begin{array}{c}\text { Non prise en compte des } \\
\text { zones à risques }\end{array}$ & $\begin{array}{c}\text { Bâti traditionnel en } \\
\text { déclin }\end{array}$ & $\begin{array}{c}\text { Savoir-faire en } \\
\text { déperdition }\end{array}$ & $\begin{array}{c}\text { réglementation } \\
\text { Construction traditionnel }\end{array}$ \\
\hline \multirow{2}{*}{$\begin{array}{c}\text { Figures de } \\
\text { développement }\end{array}$} & \multicolumn{5}{|c|}{$\begin{array}{l}\text { Urbain } 1 \text { : Tafilalet = Réseau urbain organisé en trois pôles (Grand Errachidia, Erfoud-Jorf-Rissani, Tinjdad- } \\
\text { Goulmima), encadrement territorial satisfaisant sur le plan quantitatif (Centres ruraux jouant un rôle déterminant } \\
\text { dans le réseau) }\end{array}$} \\
\hline & \multicolumn{5}{|c|}{$\begin{array}{l}\text { Urbain } 2 \text { : Tafilalet = Réseau urbain attractif capable d'absorber la pression démographique, assurant une qualité } \\
\text { de vie satisfaisante en besoins en équipement et services. }\end{array}$} \\
\hline
\end{tabular}

Tableau 1 - Les figures de dévelopement pour le dévelopement urbain 


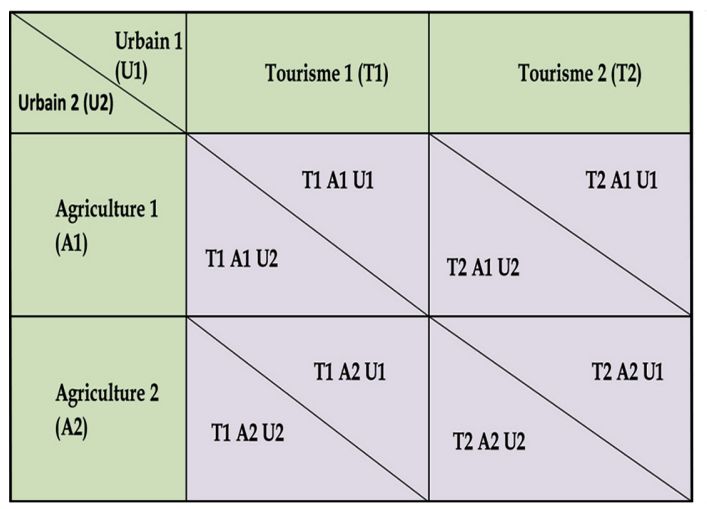

Tableau 4 - Croissement des figures de dévelopement

\begin{tabular}{|c|l|}
\hline $\begin{array}{c}\text { Futur } \\
\text { possible }\end{array}$ & \multicolumn{1}{c|}{ Déclinaison } \\
\hline $\begin{array}{c}\text { T1A1U } \\
1\end{array}$ & $\begin{array}{l}\text { Tourisme de niche, agriculture de la gestion } \\
\text { de la rareté, urbanisme tendanciel }\end{array}$ \\
\hline $\begin{array}{c}\text { T1A1U } \\
2\end{array}$ & $\begin{array}{l}\text { Tourisme de niche, agriculture de la gestion } \\
\text { de la rareté, réseau urbain attractif }\end{array}$ \\
\hline $\begin{array}{c}\text { T1A2U } \\
1\end{array}$ & $\begin{array}{l}\text { Tourisme de niche, agriculture industrielle, } \\
\text { urbanisme tendanciel }\end{array}$ \\
\hline $\begin{array}{c}\text { T1A2U } \\
2\end{array}$ & $\begin{array}{l}\text { Tourisme de niche, agriculture industrielle, } \\
\text { réseau urbain attractif }\end{array}$ \\
\hline $\begin{array}{c}\text { T2A1U } \\
1\end{array}$ & $\begin{array}{l}\text { Tourisme diversifié, agriculture de la gestion } \\
\text { de la rareté, urbanisme tendanciel }\end{array}$ \\
\hline $\begin{array}{c}\text { T2A1U } \\
2\end{array}$ & $\begin{array}{l}\text { Tourisme diversifié, agriculture de la gestion } \\
\text { de la rareté, réseau urbain attractif }\end{array}$ \\
\hline T2A2U & $\begin{array}{l}\text { Tourisme diversifié, agriculture industrielle, } \\
\text { urbanisme tendanciel }\end{array}$ \\
\hline 1 & $\begin{array}{l}\text { Tourisme diversifié, agriculture industrielle, } \\
\text { réseau urbain attractif }\end{array}$ \\
\hline T2A2U &
\end{tabular}

Tableau 5 - Présentation des futurs possibles

2.2. Phase 2: Croisement des figures de développement et détail des futurs possibles

Il s'agit de croiser chaque figure de développement de chaque enjeu stratégique avec chacune des deux figures de développements des deux autres enjeux stratégiques. Une matrice de croisement à triple entrées. Le résultat est présenté dans le tableau ci-dessous, il s'agit de huit possibilités à traiter.

2.3. Phase 3: Choix des futurs souhaitables et caractéristiques principales Dans cette troisième et dernière phase de ce processus, on procédera à l'assemblage des figures de développement par affinité stratégique. L'objectif étant de constituer des images logiques et plausibles. Ces images ne seront guère exclusives parce que la réalité est toujours composite : la prospective doit produire de l'hétérogène parce que le futur le sera sûrement, et pour que la stratégie puisse vraiment exercer des choix.

Ainsi, après la formulation des futurs souhaitables, il s'agira de trouver pour chacun d'entre eux, les actions prépondérantes, les acteurs clefs, les dangers à éviter et les lieux pertinents.

\subsubsection{Dégagement des futurs souhaitables}

Afin de faire ressortir les futurs souhaitables à partir des futurs possibles déclinés ci-dessus, le travail a consisté à apprécier l'influence (l'impact) qu'auraient ces futurs possibles sur le territoire, et ce, à travers le croisement avec les quatre piliers du développement durable 
(l'économique, le social, l'environnemental et le culturel). L'appréciation se fait sous forme d'une notation à trois valeurs (le -1 pour une influence négative, le 0 pour une influence neutre, et le 1 pour une influence positive).Le résultat est présenté dans le tableau suivant : Il ressort de la notation que sur les huit futurs possibles, trois sont souhaitables.

\begin{tabular}{|l|c|c|c|c|c|}
\hline $\begin{array}{l}\text { Futur } \\
\text { possible }\end{array}$ & Economie & Ecologie & Social & Culturel & TOTAL \\
\hline T1A1U1 & -1 & +1 & 0 & 0 & $\mathbf{0}$ \\
\hline T1A1U2 & +1 & +1 & 0 & +1 & $+\mathbf{3}$ \\
\hline T1A2U1 & +1 & -1 & 0 & -1 & $-\mathbf{1}$ \\
\hline T1A2U2 & +1 & -1 & +1 & -1 & $\mathbf{0}$ \\
\hline T2A1U1 & +1 & +1 & 0 & +1 & $+\mathbf{3}$ \\
\hline T2A1U2 & +1 & +1 & +1 & +1 & $+\mathbf{4}$ \\
\hline T2A2U1 & +1 & -1 & 0 & 0 & $\mathbf{0}$ \\
\hline T2A2U2 & +1 & -1 & +1 & -1 & $\mathbf{0}$ \\
\hline
\end{tabular}

Tableau 6 - Croissement des fururs possibles avec les piliers du dévelopement durable

tendanciel ;
Ceux-ci sont présentés ciaprès, par ordre d'importance :

- Futur souhaitable 1 :T2 A1 U2 : Tourisme diversifié, agriculture de la gestion de la rareté, réseau urbain attractif ;

- Futur souhaitable 2 : T2 A1 U1 : Tourisme diversifié, agriculture de la gestion de la rareté, urbnisme

- Futur souhaitable 3 :T1 A1 U2 : Tourisme de niche, agriculture de la gestion de la rareté, réseau urbain attractif.

\subsubsection{Caractéristiques et déclinaisons des futurs souhaitables}

«Le Tafilalet est une destination touristique à réinventer: à la recherche d'autres types de tourisme (de masse, d'affaires, scientifique, médical...) et d'activités touristiques (récréatives, sportives...) à développer, et dans lequel le tourisme de niche devient une activité revêtant un caractère secondaire. En parallèle, une agriculture (PAM, produits locaux et élevage) s'inscrivant dans la gestion de la rareté avec encouragement de la transmission des savoir-faire (formation) développé dans un statut foncier encourageant (luttant contre le morcellement et l'éparpillement du parcellaire) et une pression urbanistique maitrisée, grâce à un réseau urbain attractif capable d'absorber la pression démographique, assurant une qualité de vie satisfaisante en besoins en équipement et services». Les deux maîtres mots de ce futur sont l'attractivité et l'innovation.

\section{Actions majeures}

- Accélérer Vision 2020 : diversification de l'offre touristique ;

- Positionner le territoire à travers une démarche qualité :

- Développer des systèmes d'atténuation de la vulnérabilité des milieux sensibles ;

- Viabiliser les systèmes agricoles oasiens à travers une gestion durable de la ressource ;

- Renforcer l'attractivité du réseau urbain ;

- Un nouveau mode de gouvernance adapté ;

- Aéroport d'Errachidia à redynamiser ;
Acteurs clefs

1. ORMVAT/ABH

2. Future ADT

3. Agence Urbaine

4. Province/Collectivités

\section{Dangers / Risques}

- Dégradation de l'environnement ;

- Tarissement de la ressource en eau ;

- Crise sociale interne 
«Tafilalet est destination touristique à réinventer : à la rechercher d'autres types de tourisme (de masse, d'affaires, scientifique, médical...) et d'activités touristiques (récréatives, sportives...) à développer, et dans lequel le tourisme de niche devient une activité revêtant un caractère secondaire. En parallèle, une agriculture (PAM, produits locaux et élevage) s'inscrivant dans la gestion de la rareté avec encouragement de la transmission des savoir-faire (formation) avec statut foncier encourageant et une pression urbanistique maitrisée, à travers un réseau urbain organisé en trois pôles (Grand Errachidia, Erfoud-Jorf-Rissani, TinjdadGoulmima), dont l'encadrement territorial est satisfaisant sur le plan quantitatif (Centres ruraux jouant un rôle dans le réseau urbain).»

Actions majeures

- Accélérer Tourisme 2020 : surtout le rural ;

- Positionner le territoire à travers une démarche qualité (Tourisme + agriculture);

- Viabiliser les systèmes d'atténuation de la vulnérabilité des milieux sensibles ;

- Parachever la mise à niveau des centres (ruraux et urbains) de la province ;

\begin{tabular}{|l|l|}
\hline Acteurs clefs \\
1. Future ADT \\
2. ORMVAT/ABH \\
3. Province/Collectivités \\
\hline Dangers / risques \\
\hline - $\quad$ Réseau urbain freinant le développement ; \\
- Concentration localisée du tourisme sur les \\
sites classiques
\end{tabular}

Tableau 8 - Futur souhaitable 2: Tafilalet, territoire de la diversification maitrieée

« Le Tafilalet est une destination touristique à fort potentiel naturel et culturel à reconfirmer, évoluant dans le tourisme de niche, et dans lequel les autres types de tourismes se développement à leurs rythmes. En parallèle, une agriculture (PAM, produits locaux et élevage) s'inscrivant dans la gestion de la rareté avec encouragement de la transmission des savoir-faire (formation) ainsi qu'un statut foncier encourageant (luttant contre le morcellement et l'éparpillement du parcellaire) et une pression urbanistique maitrisée, grâce à un réseau urbain attractif capable d'absorber la pression démographique, assurant une qualité de vie satisfaisante en besoins en équipement et services». Mais la résilience prendra dans cette configuration une forme urbaine.

Actions majeures

- Renforcer l'attractivité du réseau urbain ;

- Viabiliser les systèmes agricoles oasiens à travers une gestion durable de la ressource ;

- Positionner le territoire à travers une démarche qualité (Tourisme + agriculture) ;

- Renforcement du tourisme de niche dans le réseau urbain.
Acteurs clefs

1. Province/Collectivités

2. Future ADT

3. ORMVAT/ABH

Dangers / Risques

- Déséquilibre démographique des oasis ;

- Développement anachronique des secteurs productifs.

Tableau 9 - Futur souhaitable 3: Tafilalet, territoire résilient

\section{Conclusion}

En somme, pour que les oasis de Tafilalet soit au rendez-vous avec le futur, ils sont appelés à devenir des territoires apprenants et innovants. Défi incontournable pour élaborer et mettre un œuvre un projet de territoire. Cela peut se matérialiser par la promotion de la diversité économique. L'activité touristique est devenue ces dernières années la locomotive de développement du Tafilalet, la vision Tourisme 2020 est venue pour appuyer cette tendance, notamment avec la diversification de l'offre. Pour ce qui est de l'agriculture, la remarque majeure que l'on peut tirer de l'exercice est que 
le Tafilalet aura à traiter avec les extensions agricoles hors oasis, les cultures intensives, la surexploitation de la nappe et l'accélération du tarissement de la ressource en eau. Le développement urbain, quant à lui, jouera un rôle déterminant dans le développement futur de ce territoire, vu que c'est au réseau urbain qu'incombera la mission difficile d'absorber le flux migratoire, et ce, par son attractivité et son organisation fonctionnelle d'une part, ainsi que le développement de ses reproductions sociales, simple et élargie, d'autre part, assurant in fine une qualité de vie meilleure, notamment en besoins en équipement et services.

$\mathrm{Au}$ terme de cet exercice de prospective territoriale, le parti pris méthodologique est d'offrir aux acteurs locaux du Tafilalet la possibilité de choisir la trajectoire de développement la plus appropriée. Il s'agit d'une vision de développement territorial qui peut se lire à travers trois futurs souhaitables, pris séparément ou combinés dans tous les sens. En contribuant à l'élaboration collective d'une vision stratégique pour le territoire, cet outil d'innovation sociale et territorial participe à la modernisation de l'action publique, à promouvoir un nouveau fonctionnement des organisations territoriales et à une nouvelle gouvernance territoriale. La réflexion prospective aide ainsi à stimuler l'intelligence territoriale afin d'éclairer les choix stratégiques de planification et à appréhender de façon plus globale les problématiques de sauvegarde de notre patrimoine national.

\section{Bibliographie}

Durance P., Godet M., Mirénowicz P., Pacini V. (2007), “La prospective territoriale : Pour quoi faire ? Comment faire ?, Cahiers du LIPSOR, Série Recherche $n^{\circ} 7(2008)$, CNAM, Paris, France

Jouvenel (DE) H., (2004), "Invitation à la prospective. An Invitation to Foresight, Editions Futuribles, coll. "Perspectives

Lmariouh, A., (coord.) (2007), “La prospective territoriale: démarche méthodologique. Ministère de l'Aménagement du Territoire de l'Eau et de l'Environnement, Coopération Française, Rapport

Lmariouh, A. (2014), “La prospective et l'Afrique subsaharienne, Revue Marocaine des Etudes Africaines, $n^{\circ} 1$, Université Mohamed V-Rabat

Lmariouh, A. (2014), "La prospective au Sahara marocain, Encyclopédie du Maroc : spécial Sahara, n²6 - Rabat

Lmariouh, A., (coord.) (2014), “Tafilalet 2040 : vision de développement territorial , Ministère de l'Urbanisme et de l' Aménagement du Territoire, Rapport

Loinger, G. (2004), “La prospective régionale, de chemins en desseins : Neuf étude 
de cas en France et en Europe, Editions de l'Aube - Datar Ministère de l'Habitat, de l'Urbanisme et de la Politique de la Ville, Conseil Régional de Meknès-Tafilalet (2012), "Schéma Régional d’Aménagement du Territoire (SRAT) de la région Meknès-Tafilalet, Rapport Ministère de l'Aménagement du Territoire, de l'Eau et de l'Environnement, Direction de l'Aménagement du Territoire (2004), “Stratégie Nationale d'Aménagement et de Développement des Oasis, Rapport Ministère de l'Intérieur, Province d'Errachidia, Agence Internationale de Coopération Japonaise (JICA) (2009), "Plan Provincial de Développement Rural (PPDR) , Rapport Stevens, J-f. (2000), “Petit guide de prospective Nord-Pas-de-Calais 2020, Editions de l'Aube 